GiSELA NAEGLE

\title{
Französische Gemeinwohldebatten im 15. Jahrhundert
}

Wie in anderen europäischen Ländern und im Reich spielte die Vorstellung des Gemeinwohls auch in spätmittelalterlichen Debatten in Frankreich eine wichtige Rolle. „Gemeinwohl" läßt sich für Frankreich allerdings ebensowenig wie in anderen in diesem Band beschriebenen Fällen auf einen einzigen Begriff oder eine einzige terminologische Ausprägung reduzieren, die das Konzept „Gemeinwohl“" zum Ausdruck bringt. Anhand von Beispielen aus der französischen Reformdebatte ${ }^{1}$ am Ende des 14. und Anfang des 15. Jahrhunderts sollen deshalb zunächst einige terminologische und inhaltliche Varianten solcher Diskussionen vorgestellt werden, bevor sich die Darstellung zwei Teildebatten als Beispielen zuwendet.

Das erste Beispiel betrifft das Verhältnis von Städten und Zentralgewalt, das zweite beschäftigt sich mit dem Parlement und seiner Rolle als „Hüter“ des Gemeinwohls. Dabei wird die Darstellung der Bedeutung des Gemeinwohlkonzeptes als einer für die argumentativen Auseinandersetzungen des Spätmittelalters grundlegenden theoretischen Vorstellung im Vordergrund stehen.

"Gemeinwohl" wird deshalb zunächst in einem weiten Sinn als ein abstraktes theoretisches Konzept verstanden, als die Vorstellung, eine bestimmte Handlung, Verhaltensweise, Entscheidung oder Argumentation liege im allgemeinen Interesse einer bestimmten größeren Gemeinschaft. Der Hintergrund, auf den diese abstrakte Vorstellung hier bezogen werden soll, besteht in argumentativen Auseinandersetzungen wie z.B. Prozessen, aber auch in Schriften aus dem Bereich des allmählich entstehenden öffentlichen Rechts und des „Verfassungsdenkens“ im Kontext der sich entwickelnden Staatlichkeit.

\section{Reformdebatten im späten 14. und frühen 15. Jahrhundert}

Legt man eine solche „Gemeinwohlvorstellung“ zugrunde, zeigt sich schnell, daß die Terminologie mit der das dahinterstehende Konzept zum Ausdruck gebracht wird, sehr

\footnotetext{
1 Vgl. allgemein zur französischen Reformdebatte: Bulst, 2000, S. 115-132; Contamine, S. 145-156, Cazelles, 1962, S. 91-99
} 
verschieden sein kann. Auch der jeweilige „Bezugspunkt“ ist unterschiedlich. Ein Beispiel: In Parlementsprozessen war naturgemäß die Vorstellung vom Gemeinwohl aufs engste mit dem Bereich des Rechts verknüpft. Diese Debatte war fast ausschließlich weltlich bestimmt und fand auch ihren dementsprechenden terminologischen Ausdruck. "Gemeinwohl" wurde hier vor allem im Hinblick auf den entstehenden Staat, das Royaume, den König und seine Rechte oder die Rechte von Institutionen und Untertanen formuliert. Typische Formulierungen dieses Gemeinwohlverständnisses waren das „,bien de la chose publique“ / ,bien publique“, ,,interest publique“ oder der „prouffit de la chose publique" usw. Besonders auffallend war dabei der häufige Gebrauch des Adjektivs ,publique“, das auf die „öffentliche“ Sphäre verwies, oder die Verwendung von „chose publique“. In anderen Schriften und nichtjuristischen Texten trifft man dagegen ganz andere Formulierungen an, die sich zwar auch dem Oberbegriff "Gemeinwohl“" zuordnen lassen, die allerdings einen völlig anderen Hintergrund und auch einen anderen Adressatenkreis aufweisen. Im Fall der Parlementsdiskussion war der „Adressatenkreis“ derjenigen, deren allgemeinem Wohl etwas dienen sollte, in der Regel die Gesamtheit der Bewohner des Royaume oder von Personen und Institutionen, die in irgendeiner Form deren echte oder vermeintliche Interessen repräsentierten. Im Falle der anderen Gemeinwohldebatten konnte dieser Bezugspunkt ganz anders und wesentlich umfassender sein.

Im folgenden soll am Beispiel ausgewählter Autoren verdeutlicht werden, welche Erscheinungsformen die spätmittelalterliche Gemeinwohldebatte in Frankreich annehmen konnte. Es kann sich hierbei natürlich nur um einen kleinen Ausschnitt handeln. Dabei liegt der Schwerpunkt der verwendeten Beispiele auf den Schriften von Philippe de Mézières, Christine de Pizan, Juvénal des Ursins ${ }^{2}$ und einigen kürzeren Reformschriften. Bei den genannten Autoren handelt es sich um Teilnehmer der zeitgenössischen Debatten, die durch ihre Person oder Funktion in relativ enger Verbindung zum französischen Hof standen, aber dennoch auch kritische Beobachter ihrer Zeit waren. Philippe de Mézières war z.B. zeitweise Conseiller von Charles V. und Prinzenerzieher. Er ist jedoch auch deshalb als Autor interessant, weil er weit gereist war und durch seine Erfahrungen als Chancelier von Zypern und den Aufenthalt an der Kurie von Avignon auch über Erfahrungen aus anderen Bereichen der damaligen Welt verfügte, die seinen Horizont im wortwörtlichen Sinn erweiterten: Das Anliegen seines „Songe du Vieil Pèlerin“ ist nicht nur die Reform Frankreichs, sondern letztlich eine „Weltreformation“, was sich äußerlich schon daran zeigt, daß dieser „Traum“ die Form einer Weltreise annimmt. Auch sein Engagement für einen neuen Kreuzzug weist über Frankreich hinaus. ${ }^{3}$ Christine de Pizan interessiert hier vor allem als Autorin mehrerer Werke mit fürstenspiegelartigen Zügen, auf deren Bedeutung bereits Krynen hingewiesen hat. ${ }^{4}$ Dabei ergibt sich die Bedeutung stark aus den Entstehungsbedingungen der Werke: Das bekannteste Werk Christines, „Le Livre des fais et bonnes meurs du sage roy Charles V“ war eine „Auftragsarbeit“ für den Herzog von Burgund und sollte mit seiner idealisierten Darstellung Charles V. (als positivem Beispiel) der Ausbildung des Dauphin dienen. Ein weiteres Werk, „Le Livre du corps de policie“, be-

Zur Person vgl. Thoss 1991, Bd.5, Sp. 640.

3 Zur Person vgl. Richard 1991, Bd.6, Sp. 593 und Bell 1955, S.10ff.

4 Vgl. Krynen 1993, S. $200 f f$. 
schäftigt sich ebenfalls mit der richtigen Art zu regieren. In beiden Fällen stützt sich die Autorin direkt und indirekt stark auf "Vorbilder" wie Aegidius Romanus oder Valerius Maximus oder beruft sich auf Cicero, Aristoteles oder Gestalten der Antike. Sie hat bei der „Verarbeitung“ älterer Literatur offenbar häufig auf Übersetzungen zurückgegriffen, die während der Regierung Charles V. gefördert wurden. ${ }^{5}$ Ihr Werk ist daher auch in bezug auf die Rezeptionsgeschichte der antiken Philosophie interessant, entwickelt aber anders als die Schriften von Mézières keine weitreichenden innovativen Reformvorschläge.

Schriften, die sich mit der Notwendigkeit von Reformen befassen, sehen mitunter einen Zusammenhang zwischen der Reform des eigenen Landes, Frankreich und der Reform der Kirche bzw. der gesamten christlichen Welt, „reformation de la crestiente “. ${ }^{6}$ Bezugspunkt solcher Gemeinwohlvorstellungen ist daher in manchen Fällen nicht nur das französische Royaume, sondern die „Crestienté", das heißt alle Christen oder sogar die ganze noch dem Christentum zuzuführende Welt. Einige solcher Schriften wie der "Songe" von Mézières propagieren mehrere „Reformstufen“, die bis hin zu einer „Weltreformation" führen sollen: „reformation du monde" / ,reformation de tout le monde et de la crestiente", ,reformacion de tout le monde et de toute la crestiente et par espicial du royaume de France", „reformation de l'umayne generacion ". ${ }^{7}$ Der dort verwendete Gemeinwohlbegriff unterscheidet sich sowohl terminologisch als auch inhaltlich deutlich vom weitgehend säkularen "Gemeinwohl" des Parlements. Solche Autoren sprechen z.B. vom "prouffit du bien commun de la crestiente ", ${ }^{\circ}$ vom „, bien de la crestiente" / "bien commun de la crestiente" usw. Dabei fehlt meistens der in anderen Fällen durch Adjektive wie „publique“ oder den Ausdruck „chose publique“ anzutreffende Hinweis auf den staatlichen Bereich. Wie im folgenden gezeigt werden soll, ist auch die Herkunft und der Hintergrund dieser Vorstellungen anders. Allerdings gibt es zahlreiche Übergänge, so verwendet z.B. auch Mézières in seinem „Songe du Vieil Pèlerin“ durchaus Elemente aus beiden Debatten und paßt sich sprachlich-terminologisch dem jeweiligen Umfeld an. Geht es ihm um die Reform der Kirche, der Moral und der Sitten besonders im Hinblick auf einen gemeinsamen europäischen Kreuzzug, bedient er sich des Vokabulars der allgemeiner gehaltenen, durch religiöse Elemente mitbestimmten Debatte. Seine Kritik an der Justiz und den Gerichten erfolgt jedoch in der Sprache der Juristen und der Parlementsdebatte, die er zeitweise kritisiert und zu parodieren versucht. So z.B. an dieser Stelle, an der er sich über das ständige Vertagen von Prozessen und das Hinausschieben der abschlieBenden Urteile beklagt und die Juristen mit Raben vergleicht:

„Ausquelx arquemistes [Allegorie] francois ainsi vantans et plains d'oultrecuidance [...] assez clerement se puet respondre, c'est assavoir aux dessusdiz comparez au corbeau qui ne scet autre chancon fors tant seulement, cas, cras', c'est a dire , a demain, a demain', voire de la sentence difinitive de demain en demain est prolongie".

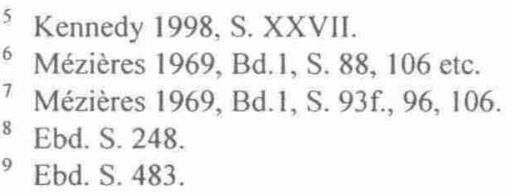


Trotzdem übernimmt er in diesem Bereich teilweise das dort verwendete Vokabular, soweit er es als Nichtjurist beherrscht. An einer anderen Stelle ahmt er den juristischen Sprachgebrauch in seiner Kritik an den Avocats nach, indem er z.B. die häufig verwendete Formel ,être en possesssion de", die ein Besitzrecht im weiten Sinn zum Ausdruck bringt, aufnimmt:

„Mais aujourduy il est tout le contraire, car les advocaz sont soutilz et malicieux, et sont en possession, comme dit est, de proposer les cautelles sustochees par lesquelles il nous convient maintesfoiz, et contre nostre volente, differer et prolonguer la sentence ". ${ }^{10}$

In diesen Teilen seiner Schrift und in dem speziell auf Frankreich bezogenen Teil kennt auch Mézières andere Gemeinwohlvorstellungen, die einen wesentlich kleineren „Adressatenkreis" betreffen: z.B. „le bien commun des Francois“, „ou prejudice du roy et du bien commun du royaume“, „contre le bien commun de France et de son roy", „au prouffit du roy et du bien commun des Francoys". ${ }^{11}$ An einigen Stellen werden auch Elemente beider Debatten miteinander verbunden, z.B. wenn es um die Person des französischen Königs in seiner Qualität als einer der wichtigsten Anführer der Christenheit geht: ,du bien et de la gloire du Blanc Faucon [frz. König] et de la crestiente“, „,[...] de travailler pour le bien commun de la generation de Adam, et par espicial de la crestiente catholique et finablement pour le bien et gloire du Blanc Faucon et par consequant du royaume de Gaulec. ${ }^{12}$ Gerade dieses letzte Beispiel zeigt, wie eng für manche Autoren das Wohl von König, Royaume, Christenheit und Menschheit insgesamt miteinander verknüpft waren. In diesem Zusammenhang bezogen sich Autoren wie Mézières, Gerson, d'Ailly, Oresme etc. auch immer wieder auf den Titel des französischen Königs als „roi très chrétien / rex christianissimus“, der ihn zu einer besonders aktiven Rolle in Angelegenheiten des Glaubens und der Kirche und bei der Beseitigung des Schismas verpflichte. $^{13}$ So heißt es dazu zum Beispiel bei Oresme in Bezug auf die Einberufung von Konzilien:

„Et as princes seculiers appartient donner a ce faire aide et confort et seureté et ce doivent vouloir et desirer souvereinement et a ce entendre tres diligenment pour le bien publique du peuple crestien [meine Hervorhebung - G.N.]. Especialment le roy de France, qui est tres catholique et vrai filz et champion de Sainte Eglise et le plus excellent de touz les princes terriens qui sunt en ce monde. ${ }^{\text {c14 }}$

Der letzte Teil dieses Zitats von Oresme stellt den französischen König aufgrund seiner besonderen Stellung als ,allerchristlichster König“ auch als den hervorragendsten der irdischen Fürsten dar. Ähnliche Zusammenhänge sah auch Mézières. Bezüglich der Verbindung von Herrschaft, Gemeinwohl und Christentum äußert er sich nicht nur zu

10 Ebd. S. 484.

11 Ebd. S. 489, 503, 507, 521.

12 Mézières, 1969, Bd.1, S. 105, 94

13 Vgl. Krynen 1993, S. 373-375.

14 Oresme 1970, S. 161. D.h. es ist eine der allerhöchsten Aufgaben der weltlichen Fürsten und besonders des französischen Königs als ,hervorragendstem aller irdischen Fürsten' im Namen des ,öffentlichen Wohls des christlichen Volkes ${ }^{`}$ den Papst bei der Einberufung von Konzilien zu unterstützen. 
Frankreich, sondern auch zu Kaiser und Reich. Verstöße gegen Gemeinwohl und Recht durch Kaiser Karl IV. waren der Grund für den Niedergang des Reiches, der mit der biblischen Prophezeiung über den Weltuntergang in Zusammenhang gebracht wird. Das Reich neigte sich Mézières zufolge mehr als jemals zuvor seinem Ende entgegen und zwar auch deshalb, weil Karl IV. von Ehrgeiz getrieben mit Hilfe des „böhmischen Goldes“ das übliche Wahlverfahren zu Gunsten seines Sohnes Wenzel manipuliert habe:

„Or pouez veoir [...] comment a present le royaume des Rommains va a declin. Et comment et plusque oncques mais a destruction et a non estre, il est enclin. Quel merveille! Car le derrain roy et empereur des Rommains meu de ambicion et en requoy ouvry les mains et par force de florins de fin or de Behaigne, qui les cuers avaricieux et tresperce et maigne, pervertit le droit commun et l'ancienne loy des esliseurs et contre l'opinion de maint preudomme et bien publique de la Crestiente [meine Hervorhebung - G.N.], fist son fils roy des royaumes; dont il est demoure desheureux et sans dominacion des Rommains, des subgiez du royaume et aussi de l'empire, dont il est pou plains". ${ }^{15}$

In einem solchen Zusammenhang sind vereinzelt auch Ausdrücke wie „bien publique de la foy catholique" etc. anzutreffen. ${ }^{16}$ Bemerkenswert ist auch, daß mitunter eine Art "Gegenbegriff" formuliert wird, bzw. der Autor das beschreibt, was eintreten wird, wenn das Gemeinwohl nicht als Handlungsmaßstab dient. In solchen Fällen ist oft von „ruyne“, „prejudice“, „dommage“, „destruction totalle“ oder „oultrage“ die Rede und zwar sowohl innerhalb der religiös geprägten als auch innerhalb der weltlichjuristischen Diskussion: Beispiele: „grant oultrage et publique dommage du peuple gallican“, „ou prejudice du roy et du bien commun du royaume“, „ou prejudice de la puissance royalle et du bien commun du royaume de France“, „le roy est pauvre et le bien commun va diminuant ", ${ }^{17}$,ou prejudice de l'utilité publique“. ${ }^{18}$

In beiden Debatten findet sich zudem oft die Gegenüberstellung Gemeinnutz - Eigennutz, wobei der Eigennutz als selbstsüchtiges, gemeinschaftsschädliches und damit unzulässiges Verhalten interpretiert wird. Dabei erscheint der „Eigennutz“ terminologisch in verschiedener Form, z.B. als ,prouffit singulier" / ,utilité privée" (im Gegensatz zur utilité publique“), als „,bien privé“" usw. In einigen Fällen erscheinen ,privater“ und „öffentlicher Nutzen" allerdings auch harmonisierbar: Bsp. ,encontre le bien publique et prive de la royalle mageste et de la communaulte des Francois" ${ }^{19}{ }^{19}$ Ein solcher Befund ergibt sich beispielsweise auch dann, wenn einzelne Personen gerichtlich versuchen, ihre Interessen gegenüber der Zentralgewalt durchzusetzen. Man versucht dann, zu zeigen, daß das eigene private Wohl in diesem konkreten Fall durchaus mit den Erfordernissen des allgemeinen Wohls zusammenfällt und die Gegenseite, die oft ebenfalls mit dem „,bien de la chose publique“ argumentiert, eine falsche Vorstellung von den daraus abzuleitenden Erfordernissen hat. Dabei spielten natürlich auch argumentationstaktische Erwägungen eine Rolle: königliche Officiers und Richter ließen sich

15 Mézières 1969, Bd.1, S. 276.

${ }^{16}$ Mézières 1969, Bd. 1, S. 253.

17 Vgl. Mézières 1969 Bd.1, S. 502, 503, 478, 504.

18 Pisan 1936, Bd.1, S. 127.

19 Mézières 1969, Bd. 1, S. 508. 
besser von der Richtigkeit der eigenen Position überzeugen, wenn man nachweisen konnte, daß die privaten Interessen des Prozeßbeteiligten auch den Interessen des Königs und des Royaume dienten und bestens mit ihnen vereinbar waren.

Ähnliche Ergebnisse wie die Untersuchung der Gemeinwohl-Terminologie bei Philippe de Mézières lassen sich bezüglich der Gegenüberstellung von Gemeinnutz und Eigennutz beispielsweise auch für Christine de Pizan, die Ordonnance Cabochienne (26-27. Mai 1413) $)^{20}$ oder die Remontrances der Universität von Paris feststellen, so zum Beispiel bei der Kritik an Officiers: „par plusieurs officiers qui n'ont pas eu l'ueil au bien de vous [des Königs] ne de la chose publique, fors seulement à leur singulier prouffit “ ${ }^{21}$ Innerhalb der Kritik an königlichen Officiers ist dieser Vorwurf, die bedenkenlose Verfolgung eigener Interessen und der Bereicherung auf Kosten des Gemeinwohls einer der am häufigsten geäußerten Kritikpunkte.

„Tournant doncques au propoz, [...] pour le bien commun du roy et de son peuple [...] les dessusdiz officiers devroyent estre retrains et regulez. Car qui vouldroit bien peser les gaiges desdiz officiers et les grans et oultrageux esmolumens qui sourdent desdiz offices oultre les gaiges ordinayres, on trouveroit une tresgrant entree et si grant revenue qu'il souffiroit pour la revenue d'un des plusgrans princes du royaume; laquelle entree superflue et dommageable au bien commun [meine Hervorhebung - G.N.] seroit mieulx seant a payer les debtes du roy et maintenir sa guerre. “22

Dies hängt damit zusammen, daß sich bereits die Vorstellung durchgesetzt hat, ein königlicher Officier sei in weit höherem Maß als andere Personen dem Maßstab des allgemeinen Wohls und der Interessen von König und Royaume verpflichtet. Die Quellen unterscheiden in dieser Hinsicht begrifflich häufig deutlich zwischen königlichen oder städtischen „Officiers" und ,personnes privées“. Für Officiers gehört es zu ihren Aufgaben, auf die Einhaltung dieser Maßstäbe zu achten und sich in dieser Hinsicht besonders vorbildhaft zu benehmen. Im Falle der Mißachtung solcher Gebote hatten die Strafen dementsprechend härter zu sein als bei „Privatpersonen“. Ein Beispiel dafür und für diese härtere Strafandrohung stammt aus einem Prozeß (1424) gegen einen ehemaligen Sénéchal der Ile de Ré, der wegen Fehlverhaltens abgesetzt worden war: ,[...] pour son mauvais gouvernement il fut debouté de l'office de senechal de l'Isle de Ré. Et dit que, veu qu'il est officier royal, de tant, a plus mespris et gravius est puniendus" ${ }^{23}$ Innerhalb solcher Diskussionen ist das Gemeinwohl jedoch nicht der einzige Wertmaßstab, sondern es wird durch weitere, ebenfalls allgemeingültige Werte ergänzt, die in manchen Fällen zur Konkretisierung mit herangezogen werden. Dazu gehören Ehre und Ruhm des Königs, Frankreichs oder des Parlements bzw. der Justiz (Honneur, Gloire) Bsp.: „,pour le prouffit, honneur et bien de vous [König] et pour la chose publique de vostre royaume“, ${ }^{24}$ „aiant l'ueil à vostre bien et honneur, à la conservacion de vostre couronne, seigneurie et l'utilité de vostre royaume“, ${ }^{25}$ "le bien et l'acroissement de son pays et de

\footnotetext{
${ }^{20} \mathrm{Vgl}$. Coville 1891.

21 In: Moranvillé 1890, S. 426.

22 Mézières 1969, Bd.1, S. 462.

${ }^{23}$ Parlement de Poitiers, Parlement criminel, Plaidoiries, in: Naegle 1999, Bd.2, S. 433.

24 In: Moranvillé 1890, S. 423.

25 In: Moranvillé 1890, S. 432.
} 
son peuple" ${ }^{26}$ weitere derartige Werte sind auch Frieden, Gerechtigkeit, Sicherheit, Rechtssicherheit, „,bonne police“, Wohlstand oder die Verteidigung des eigenen Landes.

Es erscheint angesichts terminologischer Unterschiede angebracht, für Frankreich von der Existenz verschiedener Gemeinwohldebatten auszugehen, die zwischen den beiden Extrempunkten der kirchlich-religiös-theologischen und der weitgehend säkularisierten juristischen Debatte zahlreiche Übergangsformen und Zwischenstufen aufweisen. Es handelt sich hierbei zunächst um eine Arbeitshypothese, die in Zukunft - auch im Vergleich mit Gemeinwohlvorstellungen im Reich - weiter vertieft werden soll. Die Grenzen dabei sind - wie die letzten französischen Beispiele gezeigt haben - sehr wahrscheinlich fließend. Die Bestimmung der Herkunft eines in solchen Schriften verwendeten Gemeinwohlbegriffes ist daher oft schwierig. In Bezug auf diesen Komplex werden die Fragen nach dem „Umfeld“ und der konkreten Funktion der verwendeten Gemeinwohlbegriffe bzw. die Frage nach „Substitutbegriffen" eine wichtige Rolle spielen. Dies gilt besonders für den Vergleich Frankreich - Reich. Ein kurzer Blick auf die in diesem Band von Peter Blickle beschriebenen Beispiele für die Verwendung des Begriffes „Gemeiner Nutzen", „Stadtnutz", „Landnutz" im deutschsprachigen Raum (z.B. Basel, Begründungsfigur in Tiroler Gravamina) zeigt partielle Gemeinsamkeiten mit dem spätmittelalterlichen Gebrauch solcher Begriffe durch französische Städte. In Frankreich taucht der Begriff in ähnlichen Zusammenhängen auf, so z.B. in bezug auf StraBenbau, Brückenbau, Fragen der Marktordnung und des Warenverkaufs bzw. der „Poli-

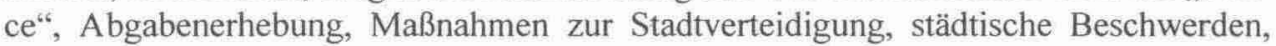
Konflikte mit Klerikern oder königlichen Officiers, Kompetenzstreitigkeiten etc. Dabei handelt es sich häufig auch um rechtliche Fragen bzw. die entsprechenden Formulierungen kommen innerhalb von städtischen Prozessen vor. Die „Fallgruppen“ und argumentativen Verbindungen, in denen sich der Bezug auf das Gemeinwohl findet, ähneln dabei auch den von Honsell für das römische Recht festgestellten Konstellationen wie z.B. Gefahrenprävention (hier v.a. militärische Bedrohung im Krieg), Rechtssicherheit, (z.B. ausdrücklich bei der Begründung der Ersitzung), Begründung von Ausnahmeentscheidungen / Appellfunktion (z.B. Abriß kirchlicher / städtischer Gebäude aus strategischen Gründen; Einforderung von Opfern angesichts der Bedrohung durch die Engländer), rechtsfortbildene bzw. ergänzende Funktion. ${ }^{27}$ Dies bedeutet jedoch noch keinesfalls, daß die Bezugnahme auf das Gemeinwohl in diesen „Fallgruppen“ direkt oder gar ausschließlich mit Hilfe römischrechtlicher Vorstellungen zu erklären wäre. Wenn es um Flüsse, Wege usw. geht, die auch als „fleuves publiques" bzw. „voies publiques“ bezeichnet werden, finden sich mitunter allerdings direkte Hinweise auf das Corpus Iuris und entsprechende römischrechtliche Vorstellungen. In ganz ähnlichen Zusammenhängen argumentieren andere Quellen allerdings manchmal mit lokalen Coutumes usw. ${ }^{28}$ Die Terminologie solcher städtischer Prozesse ist in dieser Beziehung in den

26 Pizan 1998, S. 9.

27 Für das klassische römische Recht, siehe Honsell 1978, S. 105ff., 114, 116, 122, 124, 127, 129-133.

28 Die Frage der Herkunft derartiger Begründungen aus römischem Recht, Kaiserrecht, Coutumes, Lehnsrecht etc. erörtert z.B. Leyte 1996, S. 169f. Dort finden sich auch Beispiele für verschiedene im Spätmittelalter verwendete „Begründungsmethoden“ für königliche Rechte an Wasserläufen, Ufern, Wegen etc. 
ersten Jahrzehnten des 15. Jahrhunderts in Frankreich sehr einheitlich: Man spricht fast immer vom „,bien de la chose publique“ /, ,interest de la chose publique“ / ,proufit publique" etc. Der Nutzen der eigenen Stadt wird dabei oft als Bestandteil dieses umfassenderen „Gemeinwohls" dargestellt. „Bien de la ville“ und „bien de la chose publique“ werden also - auch aus taktischen Erwägungen - bei der Vertretung der städtischen Interessen mit dem Wohl von König und Royaume harmonisiert. Angesichts zahlreicher gemeinsamer Interessen z.B. im Bereich der Verteidigung während des Hundertjährigen Krieges war dies auch in vielen Fällen möglich. Wie dieser letzte Bereich zeigt, scheint die sprachliche Formulierung der jeweiligen „Gemeinwohlvorstellung“ nach derzeitigen Erkenntnissen wichtige Hinweise zu bieten. Spricht ein Autor vom „bien commun“, geschieht dies oft im Kontext mit Verweisen auf kirchlich-theologische Autoren oder wiederentdeckte antike Autoren wie Aristoteles, Cicero etc. In derartigen Texten werden oft auch ganze Passagen oder Beispiele für ein besonders exemplarisches, positives Verhalten im Sinne des Dienstes an der Gemeinschaft übernommen. Teilweise findet dabei eine Umdeutung und Verschmelzung mit eigenen, zeitgenössischen Vorstellungen statt. So verweist Christine de Pizan z.B. auf die durch Valerius Maximus vermittelte Geschichte des „noble chevalier" Attilius Regulus, der den ,proufit de la chose publique“ höher schätzte als sein eigenes Leben und daher einen ihn begünstigenden Gefangenenaustausch ablehnte. ${ }^{29}$ Die Formulierung ,, bien de la chose publique“ / ,prouffit publique" etc. verweist eher auf die, juristische Debatte“. Auch hier lassen sich Impulse aus den anderen Bereichen jedoch nicht ganz ausschließen. Zu einer ähnlichen Schlußfolgerung kommt z.B. auch Miethke für Theoretiker des 14. Jahrhunderts: „Bei ihnen vereinigten sich tendenziell die verschiedenen Sprachangebote der einzelnen Universitätsdisziplinen, der,Leitwissenschaften' [...] zu einer wenn nicht geschlossenen, so doch integrativen theoretischen Bemühung “ ${ }^{30}$ Dies gilt besonders für den Bereich der Gemeinwohldebatten, zumal der Kreis derjenigen, die für den König als Officiers tätig waren, die an der Wiederentdeckung der antiken Autoren im sog. Frühhumanismus bzw. an den von Charles V. veranlaßten Übersetzungen partizipierten, die universitäre oder geistliche Funktionen inne hatten, oder die für den König mit diplomatischen Missionen - auch zu den Konzilien - tätig waren, oft personell übereinstimmte. Ein Beispiel hierfür ist die Familie von Juvénal des Ursins. Der hier mehrfach als Autor von Traktaten erwähnte Autor Jean II Juvénal des Ursins, Doktor beider Rechte, war im Laufe seines Lebens z.B. Erzbischof von Reims, Bischof von Beauvais und Laon und 1431-32 französischer Gesandter in Rom, er war aber auch zeitweise königlicher Conseiller, Maître des Requêtes de l'Hôtel du Dauphin und Avocat général du Roi am Parlement von Poitiers. ${ }^{31}$ Jean Juvénal vertrat also "gallikanische“ kirchliche Interessen und entstammte einer Familie, die zahlreiche königliche Officiers und Parlementsmitglieder hervorgebracht hatte. So war sein Vater z.B. unter anderem bereits Conseiller am Châtelet und Conseiller und Avocat général am Parlement gewesen. ${ }^{32}$ Jacques, einer der Brüder des Autors, war 1447 bis 1449 als Diplomat im Auftrag des französischen

\footnotetext{
${ }^{29}$ Pizan 1998, S. 76.

${ }^{30}$ Miethke 2000, S. 301.

${ }_{31}$ Müller 1990, Bd.1, S. 393-414, Bd.2, S. 962.

32 Müller 1990, Bd.1, S. 394.
} 
Königs in Lyon, Genf, Lausanne und Rom an der Beendigung des Basler Schismas beteiligt. Guillaume, ein weiterer Bruder, wurde 1445 französischer Chancelier. ${ }^{33}$ Unter derartigen Bedingungen wirkten sich natürlich auf einen Autor wie Juvénal des Ursins beide Traditionen - die kirchliche, aber ganz besonders auch die weltlich-parlamentarische Tradition - aus. Für die Gemeinwohldebatten und die Entwicklung der politischen Theorie im spätmittelalterlichen Frankreich spielten solche relativ geschlossenen Milieus, wie z.B. das des Parlements mit seinen „Parlementsfamilien“, die über mehrere dort tätige Mitglieder verfügten, eine wichtige Rolle. Die jeweilige Ausprägung der Gemeinwohldebatte ist daher sowohl von ihren Trägern und deren Ausbildung und derzeitiger Funktion, als auch vom Zweck und den Entstehungsbedingungen der jeweiligen Texte abhängig.

\section{Gemeinwohl im Verhältnis von Städten und Zentralgewalt}

Die Gemeinwohldiskussion in argumentativen Auseinandersetzungen zwischen Städten und Zentralgewalt ist zugleich ein weiterer Beleg für den wechselnden „Adressatenkreis" von Gemeinwohlkonzepten. Mitunter halten die Städte ihren Prozeßgegnern oder im Extremfall auch dem König und seinen Vertretern ein Art „städtisches Gemeinwohl“ entgegen, das auf den Kreis ihrer jeweiligen Bürger- und Einwohnerschaft bzw. auf die dann personenähnlich verstandene Stadt bezogen ist. Derartige Vorstellungen gehen teilweise im Kern auf Theorien der oberitalienischen Juristen wie z.B. Bartolus' „Tractatus de regimine civitatis“ zurück: „Sed tota civitas est una persona et unus homo artificialis et ymaginatus [...] $]^{\text {“ }}{ }^{34}$ Man spricht dann vom „bien de la ville“ oder geht sogar so weit, zu argumentieren, die Stadt sei eine eigene „res publica“ mit entsprechenden aus dieser Qualität abgeleiteten Rechten, die auch dem König entgegengehalten werden könnten. Der letzte Fall ist allerdings aufgrund einer durch die äußeren Zeitumstände des Hundertjährigen Krieges herbeigeführten weitgehenden Interessenidentität relativ selten. (Verteidigungsnotwendigkeit und Zwang zur Solidarität gegenüber dem gemeinsamen Feind England). Generell läßt sich in diesem Bereich feststellen, daß sowohl die Städte ihren Bürgern gegenüber als auch König und Zentralgewalt im Verhältnis zu ihren Untertanen im allgemeinen und den Städten im besonderen auf die Gemeinwohlvorstellung zurückgreifen, um Eingriffe in deren Rechte zu begründen. Beispiele dafür sind: Enteignungen, Abrißmaßnahmen aus strategischen Gründen, Zerstörungen von Vorstädten, die dem Feind Deckung bieten könnten, Rechtfertigung von Opfern wie Abgabenbelastungen, Zwang zu Wachdiensten oder Mitarbeit an der Stadtbefestigung, Kriegsdienst usw. In allen diesen Zusammenhängen argumentiert man mit dem „bien de la chose publique“. Dabei wird dieser Begriff oft aufs engste mit den Interessen des Königs als Person verknüpft. Er ist also oft sowohl abstrakt auf Royaume und entstehenden Staat bezogen als auch personal zu verstehen, ohne daß man dies als Ausweis von Rückständigkeit in der Begriffsentwicklung deuten könnte. Denn solche Formulierungen finden sich immer wieder im Zusammenhang mit terminologisch sehr hochent-

33 Vgl. Müller 1990, Bd.1, S. 395f.

${ }^{34}$ Bartolus 1983, S. 154. 
wickelten grundlegenden Erörterungen königlich-staatlicher Rechte oder auch der „souverainetéc. 35

$\mathrm{Zu}$ Recht hat Winfried Eberhard bereits auf die Verwendung des Begriffs als ,legitimatorisches Leitmotiv gesetzgeberischen und politischen Handels in den Ordonnanzen der französischen Könige“" hingewiesen. ${ }^{36}$ Ähnliches gilt auch für Privilegienerteilungen und Formularsammlungen der königlichen Kanzlei. Die Berufung auf das Gemeinwohl war jedoch kein Monopol des Königs, seiner Officiers und der Institutionen. Auch die Städte gegenüber der Zentralgewalt und die Bürger gegenüber den Städten beriefen sich auf das Gemeinwohl, das sie richtiger zu erkennen glaubten als die jeweilige Gegenseite. Dabei konnte es auch zu einer „oppositionellen“ Verwendung des Begriffes kommen, wie z.B. bei den „Cabochiens" 1413, während der „Ligue du Bien Public" oder bei anderen städtischen Unruhen. ${ }^{37}$ Eine in Konfliktfällen häufig verwendete Argumentationsfigur war hierbei die Gegenüberstellung der als eigensüchtig und damit verwerflich interpretierten Interessen des Einzelnen oder der einzelnen Stadt und des allgemeinen Wohls der jeweiligen Gemeinschaft (Stadt, Royaume, Christenheit). Es findet sich dann stets die Aussage, das Wohl des Einzelnen oder der kleineren Einheit müsse hinter dem als übergeordnetes höherwertiges Interesse verstandenen Gemeinwohl zurücktreten. Prinzipiell mußte aber keineswegs immer ein Spannungsverhältnis oder ein Interessengegensatz vorliegen und das Interesse des Einzelnen und der Gemeinschaft waren oft in einem übergeordneten Gemeinwohl integrierbar. Dieser letzte Aspekt zeigt sich bei der Bemühung um neue Privilegien, z.B. für die Abhaltung von Messen, für die Erlaubnis zur Erhebung neuer Abgaben, beim Bemühen um Ermäßigung oder Überlassung bestimmter Abgaben. Weitere derartige Fälle sind die Bitte um königliche Hilfe z.B. bei der Stadtverteidigung, oder das Streben, Sitz königlicher Institutionen zu werden. Hierbei wurde stets versucht, nachzuweisen, daß das, was dem Wohl der Stadt diene, auch im Interesse von König und Royaume liege. Auch hier findet sich immer wieder der Verweis auf das ,bien de la chose publique“. Im Hinblick auf das Ziel der jeweiligen Bemühungen werden dann allerdings konkrete Vorteile aufgezählt, die König und Royaume durch die Erteilung der Privilegien oder die geforderte Hilfeleistung zu erwarten haben. Der Begriff "Gemeinwohl“ wird hier also auf den jeweiligen Einzelfall bezogen und dadurch konkretisiert, es fehlt jedoch eine grundsätzlich-theoretische Definition. Diese Frage bleibt in der Regel offen und wird durch den bloßen Hinweis auf das „bien de la chose publique“, „bien commun“, ,interest publique“ usw. oder auf ergänzende Werte wie das „,bien de justice“, die „équitéc etc. ersetzt. Das jeweilige Ziel der verlangten oder zu unterlassenden Handlung und die dadurch zu erwartenden Vorteile oder Nachteile werden allerdings benannt. Es ergibt sich bei Prozessen in der Regel bereits aus dem Ziel der Klage. Es handelt sich bei dem Verweis auf das Gemeinwohl also um einen jeweils inhaltlich durch die jeweilige Situation und den Kontext der Verwendung bestimmte Formel. Wobei insbesondere die schädlichen Auswirkungen des nicht mit dem Gemeinwohl in Einklang stehenden Verhaltens immer

${ }^{35}$ Kurzfassung einiger Ergebnisse der Untersuchung der städtischen Prozesse vor dem Parlement von Poitiers. Ausführlich dazu: Naegle, 1999, Bd.2, Kapitel 7-8, S. $298 \mathrm{ff}$.

36 Eberhard 1985, S. 199.

${ }^{37}$ So auch Eberhard 1985, S. 208-212, 203. 
wieder - und im Hinblick auf den Argumentationszweck mitunter sehr drastisch - beschrieben werden. Sie können z.B. im völligen Ruin der Stadt oder Landschaft, des Royaume, der Christenheit oder im Extremfall - bei Aussagen mit religiösen Aspekten - im Verlust von Kriegen oder im Weltuntergang und der Vernichtung der Menschheit oder der ewigen Verdammnis bestehen.

Hinsichtlich der Gemeinwohlargumentation in städtischen Prozessen stellt sich die Frage nach der Herkunft des verwendeten Gemeinwohlbegriffes, die im Einzelfall sehr schwer und mitunter gar nicht zu beantworten ist. Ein Grund dafür besteht darin, daß ein wesentlicher Teil der überlieferten Quellen, die Einblicke in derartige Debatten erlauben, Prozeßregister sind. Die eigentlichen Akten existieren in der Regel - von seltenen Glücksfällen abgesehen, in denen Prozeßbeteiligte Unterlagen zu ihren Verfahren gesammelt haben - nicht mehr. Die erhaltenen Prozeßregister und Accords bieten nur eine verkürzte Form der Argumentation, die zudem aus den rein chronologisch geordneten Registern erst zu einem möglichst vollständigen Bild des jeweiligen Prozesses zusammengesetzt werden muß. In den meisten Fällen führt dieses Verfahren dennoch nur zu mehr oder weniger ausführlichen Prozeßfragmenten. Hinzu kommt, daß die Register der Plädoyers aus dem Gedächtnis angefertigt sein dürften und nur selten eindeutig identifizierbare Zitate, etwa aus dem Corpus Juris oder aus Coutumes enthalten. Oft sind die wiedergegebenen Zitate nicht mehr identifizierbar, weil verkürzt, verändert oder nur mit sehr allgemeinen Hinweisen versehen wie z.B. auf die „anciens“ oder die allgemeine Üblichkeit „de si long temps qu'il n'est memoire du contraire“.

In vielen Fällen wird auch eine ganze Sammlung verschiedener Autoritäten und Rechtsbereiche in sehr allgemeiner Form aufgezählt. Ähnliches gilt mitunter auch für theoretische Schriften, wie hier z.B. bei Christine de Pizan: „[...] comme il soit de droit escript et loy que tous princes naturelx puissent user et prendre sus leur subgiez en certains cas necessaires comme pour soustenir les guerres et deffenses du reaume et du bien commun et autres cas". ${ }^{\text {. }}$

Hier wird also explizit auf das geschriebene Recht und die Gesetze verwiesen, aber ohne die Quellen genauer anzugeben. Derartige Pflichten ließen sich natürlich aus dem geschriebenen Recht, aus Coutumes oder königlichen Ordonnances ableiten. Solche Pflichten finden sich aber auch traditionell unter den lehnrechtlich begründeten sog. "Quatre cas". Worauf sich eine solche Aussage bezieht, muß damit offen bleiben. Ein weiteres Problem bei der Identifizierung von Quellen besteht darin, daß selbst dem römischen Recht ähnliche Verjährungsfristen oder Bestimmungen nicht direkt daraus abgeleitet sein müssen: Sie könnten auch aus romanisierten Coutumes stammen, oder es könnte eine zufällige Ähnlichkeit vorliegen.

Die Interpretation erschwerend kommt hinzu, daß sich für juristische Tatbestände auch das Problem des Anachronismus stellt, d.h. es besteht die Gefahr Rechtsinstitute späterer Zeiten „zurückzuinterpretieren“ oder - gerade wenn ausgeprägte Ähnlichkeiten auftreten - eine Art lückenloser Vorgeschichte konstruieren zu wollen. In diesem Zusammenhang sind terminologische und inhaltliche Aspekte eng und komplex miteinander verzahnt und die „Anachronismus-Frage“ ist im Einzelfall schwierig zu beantworten.

38 Pizan 1936, Bd.1, S. 69. 
Einer etwas älteren, aber als Ergänzung anderer Theorien immer noch verwendeten juristischen Definition - der sog. „Interessentheorie“ zufolge gehört ,eine Norm, die überwiegend dem Interesse der Allgemeinheit dient, zum öffentlichen Recht, eine Norm, die überwiegend dem Interesse von Einzelnen dient, zum Privatrecht" . ${ }^{39}$ Handelt es sich hierbei um eine anachronistische Definition? Zu Recht ist immer wieder darauf hingewiesen worden, daß der Begriff ,ius publicum“ erst in der frühen Neuzeit und während der allmählichen Entstehung des modernen Staates schärfere Konturen erhält. ${ }^{40}$ Ein Blick auf die Geschichte: Bereits das römische Recht kennt den Ausdruck „ius publicum“, im Unterschied zum ,ius privatum“. In den Digesten heißt es dazu: „Publicum ius est quod ad statum rei Romanae spectat, privatum quod ad singulorum utilitatem: sunt enim publice utilia, quaedam privatim“ (D.1.1.1.) Mittelalterliche gelehrte Juristen kannten diese Definition und wandten sie auch an. So spielten für die Frage der Abgrenzung, ob es in einem Prozeß aus den ersten Jahrzehnten des 15. Jahrhunderts - Streitigkeiten im Zusammenhang mit der Vergabe von Metzgerständen in La Rochelle - um „öffentliche“ oder ,private“ Interessen ging, die eben zitierten Grundsätze eine wichtige Rolle. Bemerkenswert ist dabei der direkte Verweis auf das ,interest de la chose publique" und die Beteiligung bzw. Nichtbeteiligung königlicher Officiers am Proze $\beta$ und seinen Vorstufen als Unterscheidungskriterium der beiden Fälle:

„[...] dit que les demandeurs n'ont corps ne college [...] ainsi ne sont ni la plus grant ne la plus saine partie, ne de chose dont est question par eulx n'y a interest a la chose publique, ne il n'y a plaintif, ne les procureur ne les gens du roy de pardela n'en ont riens escript et n'est pas samblable au fait des bouchers de Bourges qui fut a la complaincte des habitans et par deliberacion du Conseil du roy $[\ldots]^{*}{ }^{41}$

Die Abgrenzung der verschiedenen Rechtsgebiete in den Digesten ist nicht die einzige, auf die mittelalterlichen Autoren zurückgreifen konnten. Auch das kanonische Recht beschäftigt sich mit dieser Frage und der Unterscheidung und Abgrenzung von „ius naturale“, ,ius civile“ und ,ius gentium" usw. Im Corpus Iuris Canonici heißt es beispielsweise: „Ius publicum est in sacris et sacerdotibus et magistratibus“ (Dist.1, C.XI.). Sowohl die römisch-rechtliche als auch die kanonistische Abgrenzung der verschiedenen Rechtsbereiche werden explizit oder implizit viel verwendet und miteinander vermischt. Ein Beispiel dafür aus den späten 1570er Jahren ist die Definition des ,ius publicum“ in der „Juris universi distributio" von Jean Bodin, der selbst römisches Recht in Toulouse gelehrt hatte und als Avocat am Parlement von Paris tätig gewesen war. Hier lassen sich deutlich Elemente aus beiden älteren Definitionen erkennen:

\footnotetext{
„Publicum est, quod publicam utilitatem consectatur, cujusmodi sunt, Sacra tueri, Leges jubere, Magistratus creare, Consilium de Repub. capere, Bellum indicere ac finire, Poenas ac praemia irrogare, Legis actiones exequi “. 42
}

Es läßt sich also feststellen, daß der spätmittelalterlich-frühneuzeitliche Begriff des Ius publicum sehr viel weiter gefaßt war als der heutige und unter anderem auch Rechtsbe-

39 Köbler 1986, S. 175.

40 Stolleis 1988, S. 47.

${ }^{41}$ Darstellung des Prozesses und Wiedergabe der Textstelle in: Naegle, 1999, Bd.2, S. 550.

42 Bodin, 1985, S. 20. 
reiche mitumfaßte, die heute in den Bereich der zwischenstaatlichen Beziehungen, der Diplomatie oder des Völkerrechts fallen.

Wie komplex das Verhältnis zwischen römisch-kanonischem und mittelalterlichem Recht sein kann und wie schwierig sich im Einzelfall die Zuordnung einer Stelle gestalten kann, verdeutlicht eine Aussage von Jean Juvénal des Ursins aus der „Exortation faicte au roy nostre souverain seigneur":

„[...] vous [der König] n'estes de riens subgect aux lois rommaines; vous estes empereur en vostre royaume, lequel tenés de Dieu et de l'espee et non d'aultre [meine Hervorhebung G.N.]. Et supposé que en vostre royaume on ait accoustumé de user du sens et entendement de la loy en tant qu'il touche confiscacion de corps et de biens [...], toutevoye ce n'est pas comme subgect a la loy, mais pour ce que, selon bonne justice et entendement, raison veult que ainsi soit fait. ${ }^{\text {43 }}$

Das Zitat stammt aus einem Zusammenhang, in dem es um die Beurteilung von Majestätsverbrechen, einer besonders stark römisch-rechtlich geprägten Materie, geht: Selbst hier ist der französische König nach den Vorstellungen Juvénal des Ursins' nicht an das römische Recht gebunden. Aufgrund seiner kaisergleichen Stellung innerhalb des Royaume ist er ihm nicht unterworfen. Selbst wenn es in Frankreich üblich sei, den Grundgedanken der römisch-rechtlichen Bestimmungen zu folgen, so könne dies keinesfalls auf den verpflichtenden Charakter dieser Regeln für den König zurückgeführt werden, sondern ergebe sich lediglich aus den Geboten der Vernunft und der Gerechtigkeit.

Damit verweist Juvénal des Ursins indirekt auf einen für die rechtliche Entwicklung sowohl Frankreichs als auch des Reichs zu bedenkenden Aspekt: das römischkanonische Recht war bei weitem nicht die einzige Wurzel mittelalterlichen Rechts, sondern drang erst allmählich im Zuge der sog. Rezeption in immer größere Bereiche vor, die früher durch ungeschriebenes Gewohnheitsrecht bestimmt worden waren. Dieses Gewohnheitsrecht z.B. in Form von Coutumes in Frankreich oder des ,alten Herkommens" im Reich konnte regional sehr verschieden und unterschiedlich stark entwikkelt sein. In beiden Fällen wurde es nicht völlig verdrängt, sondern galt weiterhin - oft allerdings nur subsidiär bzw. wenn seine Existenz sicher bewiesen werden konnte. In Frankreich kam es auf königliche Anordnung hin schließlich ab der zweiten Hälfte des 15. Jahrhunderts zu einer groß angelegten Welle von Coutume-Aufzeichnungen. Hinzu kommt auch, daß das römisch-kanonische Recht weder im Reich noch in Frankreich in der ,klassischen Form" rezipiert wurde, sondern vor allem so, wie es sich nach der Bearbeitung durch oberitalienische Juristen wie Bartolus und Baldus oder der Auslegung durch bestimmte Rechtsschulen französischer Universitäten darstellte. Bei dieser Bearbeitung kam es zu einer Anpassung an die mittelalterlichen Lebensverhältnisse, die die ursprüngliche Substanz mitunter erheblich veränderte. Zeitpunkt, Art und Ausmaß der Rezeption waren regional sehr verschieden. In Südfrankreich setzte die Rezeption z.B. sehr früh ein, während der Norden stark durch Coutumes geprägt blieb. Im einzelnen gesehen, gibt es hier noch zahlreiche Forschungslücken.

So stellt Gauvard für den Bereich des Strafrechts fest, es sei nach derzeitigem Kenntnisstand noch nicht möglich zu sagen, wie stark der Einfluß des römisch-kanonischen

43 Juvénal des Ursins 1985, S. 413. 
Rechts oder der Lehren von Bartolus und Baldus auf Strafurteile gewesen sei, da es noch keine Untersuchungen und quantifizierenden Inventarisierungen von diesbezüglichen Zitaten und Verweisen gebe. ${ }^{44}$ Für andere Rechtsgebiete und das Reich gilt Ähnliches, auch wenn seit einiger Zeit intensiv an solchen Fragestellungen gearbeitet wird.

Hinzu kommt, daß in späterer Zeit das Verhältnis zum römischen Recht mitunter sehr distanziert gesehen oder es teilweise völlig abgelehnt wurde. Die Gründe für die Ablehnung hängen auch mit einem allmählichen Aufschwung des Nationalgefühls bis hin zu recht extremen Formen, die man heute als „Nationalismus“ bezeichnen würde, zusammen.

\section{Das Parlement als Hüter des Gemeinwohls}

Wie bereits an anderen Beispielen deutlich wurde, kam es in Frankreich schon früh und vor allem wesentlich früher als im Reich zur Entstehung differenzierter zentraler Institutionen, die im Laufe der Zeit ein beträchtliches Eigengewicht und Eigenbewußtsein entwickelten wie z.B. das Parlement oder die Chambre des Comptes. Parallel dazu entstand - auch hier in deutlichem Unterschied zu den deutschen Verhältnissen - die neue Schicht der sog. „noblesse de robe“ eines Standes königlicher Officiers mit spezifischem Verhalten, Ethos und Zusammengehörigkeitsgefühl. Diese einigenden Faktoren lassen sich sowohl prosopographisch wie z.B. in der Arbeit von Françoise Autrand „Naissance d'un grand corps de l'État" ${ }^{\text {“5 }}$ und weiteren Untersuchungen der letzten Jahre, als auch ,inhaltlich“ anhand von Selbstaussagen aus Prozessen etc. fassen. Königliche Ordonnances bezeichneten das Parlement gelegentlich als „fons et origo justitiae totius regni““ ${ }^{46}$

Das Parlement selbst hatte ebenfalls eine sehr hohe Meinung von sich. Es nahm auf seine „Außenwirkung“ bezug und behauptete, nicht nur „subditi quin etiam extranei et sarraceni“ hätten das Parlement in seiner Funktion als oberstes Gericht („,suprema et capitalis representans regem immediate") angerufen. Auch Poitiers verwies bei seinen Bemühungen, das Parlement zu behalten, auf dessen „außerfranzösisches Ansehen“ und sprach davon, der Glanz der ,justice souveraine de ce royaume" sei in der Vergangenheit in allen christlichen Königreichen zu sehen gewesen (,la justice qui ou temps passé a esté reluisant par tous les royaumes crestiens $\left.{ }^{4}\right){ }^{47} \mathrm{Da} ß$ diese hohe Meinung durchaus einen realen Hintergrund hatte, zeigen einige bewundernde Aussagen aus dem Reich. So erwähnt z.B. der von Ranke abgedruckte „Abschiedt zwischen geistlichen Churfürsten, mit waß mittel das Rom. Reich wieder uffzubringen wäre [...]" (um 1454) auch das Parlement: „Item, das eyn gericht ordinert werde, mit eyner nemlicher zale personen von allem stade, die stediß alle sachen ußrichten, Im rechten, in glycherwyse als an dem par-

44 Gauvard 1997, S. 31f.

45 Autrand 1981.

46 Krynen 1989, S. 334, Anm. 7.

${ }^{47} \mathrm{Vgl}$. Mémoire von Poitiers, 50er Jahre des 15. Jhds., Zitat nach dem Original aus Naegle 1999, Bd.1, S. 278. 
lament zu paryß, als von alters dick gescheen ist, und man die forme davon noch woil findet". 48

Der sog. „Oberrheinische Revolutionär“ nimmt ebenfalls auf das Parlement bezug, auch wenn es in seiner kurzen Beschreibung kaum wiederzuerkennen ist:

„Dorumb noch die Franzosen sagen nach underwisung der Triereren: halten das parliment, das ist das palatium ymperiale consistorii, das ein pfaltzgraff halten soll, do der armen sin mecht beclagen. Und uff ein Tag můst iederman verfasset sin. Und wer verlor die sach, hat ers nit am gưt zu bezahlen, so strofft man im an dem lib" “ ${ }^{49}$

Zumindest der Aspekt, daß vor dem Parlement auch ,arme Leute“ zu ihrem Recht kommen sollten, war auch den Franzosen selbst wichtig, so heißt es z.B. in einem städtischen Mémoire aus Poitiers (aus den 1450er Jahren):

„Item que par ces moyens [...] la justice souveraine semble n'estre deuement administree a tous les subgietz du royaume [...] et est chose tres piteable que le povre peuple qui de son puvoir a loyaulment servy et obey aud. sr. et tousiours fera jusques a la mort soit forclox par ces moyens [die Rückkehr des Parlements nach Paris] du bien de justice". ${ }^{\text {" }}$

In der Tat fanden vor dem Parlement im 15. Jahrhundert zahlreiche Prozesse „einfacher“ Leute, von Handwerkern und dörflichen Gemeinden statt.

Obwohl also das Parlement durchaus Gegenstand der Bewunderung war, wurde es auch zur Zielscheibe von Kritik, die allerdings oft etwas milder ausfiel als für andere Institutionen und Juristen, da verglichen mit den übrigen das Parlement seine Aufgaben doch noch besser erfülle: ,il se puet dire que ce sont ceulx qui mains sont corrumpuz". ${ }^{51}$ Auch Mézières kann sich allerdings der Bewunderung für das Parlement nicht ganz entziehen, da es der direkteste Ausdruck des Lebens des Royaume sei und den König und seine Majestät repräsentiere: „c'est assavoir aux grans juges et a la haulte justice qui ou royaume pardessus tous juges representent le roy et sa royalle mageste, c'est assavoir au benoist parlement, qui par sa nature est la vie du royaume“. ${ }^{52}$ Mézières vergleicht das Parlement zusammen mit Requêtes und Enquêtes sogar mit der heiligen Dreieinigkeit und tituliert es selbst als ,heilig“, indem er vom „,benoist" oder ,saint Parlement" spricht. ${ }^{53}$ Dennoch sieht er die Lage von Justiz und Gerichten insgesamt sehr düster. Alle Mißstände wirken sich zu Ungunsten des „bien commun“ und der „chose publique“ aus. So z.B. auch die von ihm kritisierte überlange Dauer von Prozessen, besonders für weniger wohlhabende Parteien, die dadurch in Armut geraten könnten:

„si est que les subgiez du roy par longues plaidoiries, pour lesquelz ilz sont cheuz en pauvrete, n'ont de quoy il puissent servir le roy, ne faire leur devoir a leur seigneur naturel, ou prejudice de la puissance royale et du bien commun du royaume de France". 54

\footnotetext{
48 Ranke 1868, S. 12.

49 „Oberrheinischer Revolutionär" 1967, S. 255

50 Naegle 1999, Bd.1, S. 281, nach dem Original.

S1 Mézières 1969, Bd.1, S. 475.

52 Mézières 1969, Bd.1, S. $472 f$.

53 Mézières 1969, Bd.1, S. 473.

54 Ebd. S. 478.
} 
Mézières kritisiert wie einige andere Zeitgenossen heftig den Aufstieg und Einflußgewinn der Juristen, die sich zudem zunehmend in Bereiche hineindrängten, in denen sie nichts zu suchen hätten. Er wirft ihnen unter anderem die bewußte Verlängerung von Prozessen, Spitzfindigkeiten und - besonders den Avocats - zahlreiche Kunstgriffe zur Verfälschung der Wahrheit vor. Demgegenüber wird eine ,goldene" Vergangenheit idealisiert und behauptet, der Begründungsaufwand der Juristen sei verzichtbar:

„si est que les advocaz de commun cours, par sophismes et soutilz argumens, par allegacions de loix, decretalles, de costumes abusives, et divers privileges, ont acoustume par une possession acquise de destourber souvent les juges de jugier verite ${ }^{\text {". }}$. $^{5}$

Trotz der geforderten Rückkehr zu Einfachheit und Gerechtigkeit und einigen Vorschlägen, die teilweise utopische Züge aufweisen, möchte Mézières nicht völlig auf gelehrte Juristen verzichten, aber es muß dringend zu einer Verbesserung der Zustände kommen, da das Gemeinwohl völlig daniederliege:
„Mais vous autres juges, docteurs et avocaz avez tant estudie et multiplie les gloses que la subs- tance et entencion de la loy, pour le bien du commun abregiee, aujourduy en son effect est comme toute perie[...]". 56

Einige der von ihm genannten Kritikpunkte finden sich auch in anderen Reformschriften. Derartige Vorwürfe sind ein wichtiger Grund dafür, daß spätmittelalterliche und frühneuzeitliche Gerichte von der Forschung lange Zeit überwiegend negativ beurteilt wurden. $\mathrm{Zu}$ den immer wieder geäußerten Vorwürfen gehören: der immer weiter anwachsende „Rückstau“ von unerledigten Prozessen, die überlange Prozeßdauer, die geringe Entscheidungsfreude der Richter und unzulängliche Vollstreckungsmöglichkeiten bzw. die mangelnde Durchsetzbarkeit der gefällten Urteile. ${ }^{57}$

Selbst jemand wie Juvénal des Ursins, der das Parlement aus eigener Tätigkeit kannte, äußerte mitunter eine derartige Kritik. Auch hier war der Bezugspunkt immer wieder das Gemeinwohl:
"[...] et encores quant le president ordonne que on oeuvre l'uys pour plaider il n'en aura avec lui que cinq ou six, et souvent, comme l'en dit, preferent leurs besongnes particulieres ou de leurs amis au bien de la chose publique [meine Hervorhebung - G.N.]. Et dient aucuns que quant ilz vont en commissions ilz besongnent tres lachement et prolongent leurs matieres, ad fin qu'il y ait plus de jours pour plus gaignier. Et ou paiement on treuve que ilz sont bien rigoreux [...]".58

Dennoch zeigt der Blick auf das überlieferte Quellenmaterial, daß solche Vorwürfe zwar einen wahren Kern hatten, daß die Prozeßwirklichkeit und die tatsächlichen Leistungen der Gerichte aber längst nicht so schlecht waren, wie die Aussagen ihrer Kritiker vermuten lassen. Ein Teil der Kritik hängt mit sich verändernden Rahmenbedingungen wie der zunehmenden Professionalisierung und Juridifizierung der königlichen

55 Ebd. S. 464

${ }^{56}$ Ebd. S. 500. Mit seiner Kritik an Justiz und Parlement steht Mézières nicht allein. Zur Juristen- und Gerichtskritik im Songe von Mézières vgl. Krynen 1989, S. 87-111.

57 Diestelkamp 1995, S. 33, für das Reichskammergericht. Für das Parlement bzw. die Beurteilung durch die französische Forschung gilt Entsprechendes.

58 Juvénal des Ursins 1985, Bd.2, S. 335. 
Verwaltung und der Institutionen und Verfahren bis hinunter auf die untere Ebene der Städte zusammen. Daraus ergab sich ein erheblich gewachsenes Prozeßaufkommen auch im Bereich der obersten Gerichte, auf das sie sich erst allmählich einstellen mußten. In vielen Fällen trugen die Prozeßparteien, soweit ihnen diese Entwicklung dienlich war, selbst zu dieser Veränderung bei, oder sie setzten bei entsprechendem Interesse selbst alles daran, ihren Prozeß zu verschleppen.

Der gemeinsame Nenner bei der Beurteilung des Parlements ist jedoch seine Aufgabe als Hüter und Wahrer des „bien de la chose publique", die ihm sowohl nach seiner eigenen Meinung als auch in den Augen seiner Kritiker zukam. Diese Aufgabe erfüllte das Parlement durchaus auch gegenüber dem König, wenn es „Remontrances“ vorbrachte oder die Registrierung von Privilegien verweigerte, die eine Schmälerung oder einen Verlust von königlichen Rechten bedeuteten. Nicht immer war der Widerstand gegen diesbezügliche königliche Maßnahmen erfolgreich (z.B. bei Abtretungen von Teilen des domaine royal), aber dennoch gibt es zahlreiche Fälle, in denen das Parlement oder andere große Institutionen ihren Standpunkt durchsetzen konnten. Gerade in der unruhigen Zeit des Hundertjährigen Krieges und der in diesem Zusammenhang auftretenden Krisen des Königtums stellten sie einen wichtigen Faktor der Stabilisierung dar. Wie die große Zahl von Prozessen auch aus kleineren Städten und sogar dörflichen Gemeinden oder von Einzelpersonen zeigt, nahmen auch die Zeitgenossen diesen Faktor durchaus wahr. ${ }^{59}$

Gerade das Beispiel des Parlements, mit seinem hohen inner- und außerfranzösischen Ansehen, die Tatsache, daß es als Instanz rechtlicher Konfliktregelung bis auf die Ebene von Dörfern hinein zunehmend an Bedeutung gewann und der Blick auf die säkularisierte ,juristische" Debatte zeigen, daß die Bezugnahme auf das Gemeinwohl in Frankreich im Spätmittelalter stark integrative Aspekte aufwies. Zwar beriefen sich auch „Oppositionelle" und Anführer von Unruhen auf das Gemeinwohl, aber selbst hier geschieht dies in der Regel nicht in der Form, daß man eine wirklich neue, eigenständige Definition der wünschenswerten Zustände oder eines „,anderen“ Gemeinwohls entwirft. Vielmehr orientieren sich die Forderungen oft eher rückwärtsgewandt an einer idealisierten Vergangenheit und man beansprucht lediglich, den situationsbezogenen Erfordernissen des Gemeinwohls besser gerecht zu werden. Ein Beispiel für diese Haltung findet sich auch in Étienne Marcels Brief an die Échevins von Ypern vom 28. Juni 1358, wo es zu den Zielen der erbetenen Hilfe für Paris unter anderem heißt,

„[...] par telle manière que nous tous puissions vivre en france liberté selon ce que ordené fu du temps ancien au royaume de France.“ (,,[...] so daß wir alle in ,fränkischer' Freiheit leben können, wie es in alter Zeit im Königreich Frankreich bestimmt wurde." $)^{60}$

Schaut man sich die Herkunft oder die Funktion der Verfasser von Reformschriften an, so zeigt sich, daß viele von ihnen dem König bzw. dem Hof nahestanden oder zumindest zeitweise königliche Officiers waren. Eine ganze Anzahl derer, die sich an den spätmittelalterlichen französischen Gemeinwohldebatten beteiligten, hatten also die für sie oft stark prägende Erfahrung der entstehenden "großen Institutionen" gemacht und waren durch

\footnotetext{
59 Liste von an Prozessen vor dem Parlement von Poitiers beteiligten Ortschaften, in: Naegle 1999, Bd.2, S. 635-642.

${ }^{60}$ D'Avout 1960, S. 304.
} 
die dort üblichen Argumentationsweisen beeinflußt. Auf diese Weise waren die Gemeinwohldebatten auch ,innere“ Debatten innerhalb der sich immer stärker entwickelnden Verwaltung. Hinzu kam, daß gerade diese entstehende Verwaltung in den Wirren des Hundertjährigen Krieges als Faktor der Stabilität in vielen Bereichen den mitunter abwesenden oder weitgehend handlungsunfähigen König ersetzte, sozusagen vor Ort für ihn regierte und auch ihrem Selbstverständnis nach seine Interessen wahrnahm. Hinzu kam, $\mathrm{da} ß$ in zahlreichen Städten die bedeutendsten Familien der regierenden Stadtoligarchie auch die wichtigsten königlichen Offices inne hatten und immer stärker nach solchen Funktionen und nach juristischer Bildung strebten. Auf diese Weise schloß sich der Kreis, da es so immer weniger mögliche Interessengegensätze zwischen den führenden städtischen Familien und den Vertretern der Zentralgewalt gab. Konsequenterweise beriefen sich in Prozessen dann auch beide Seiten auf das Gemeinwohl. Dabei mußte sich die jeweilige - fallbezogene - Definition nicht unbedingt grundsätzlich unterscheiden, sondern es ging lediglich um das ,,richtigere“ Verständnis derselben Sache und die daraus abzuleitenden Konsequenzen. Ein Beispiel dafür bietet in dieser Zeit die Identifizierung von Gemeinwohl und Sicherheitsinteresse bzw. der Notwendigkeit der Verteidigung gegen den äußeren Feind. Daß diese Verteidigung im allgemeinen Interesse lag, bezweifelte in der Regel keine der an einem Rechtsstreit beteiligten Parteien. Strittig war vielmehr, welche Maßnahmen erforderlich oder welche Eingriffe in eigene Rechte noch zumutbar waren. Dennoch zeigt gerade dieses letzte Beispiel, daß hier die Berufung auf das Gemeinwohl einen integrativen Appellcharakter haben konnte.

Quellen

Bartolus (1983), De regimine civitatis, in: Politica e diritto nel Trecento italiano, hg. v. D. Quaglioni, Florenz.

Bodin, J. (1985), Exposé du droit universel, Juris universi distributio, hg. v. Bl. Barret-Kriegel, Paris. Juvénal des Ursins, J. (1985), Écrits politiques de Jean Juvénal des Ursins, hg. v. P. S. Lewis, Bd.2, Paris. Marcel, É. (1960), Lettre aux échevins d'Ypres, 28 juin 1358, in: Le meurtre d'Étienne Marcel, hg. v. J. D'Avout, Paris, 1960, S. 303f.

Mézières, Ph. de (1969), Le Songe du Vieil Pèlerin, hg. v. G. W. Coopland, 2 Bde., Cambridge.

Moranvillé, H. (Hg., 1890), Remontrances de l'Université et de la ville de Paris à Charles VI sur le gouvernement du royaume (1890), in: Bibliothèque de l'École des Chartes 51, S. 420-442.

„Oberrheinischer Revolutionär“ (1967), Das Buch der Hundert Kapitel und der vierzig Statuten des sog. Oberrheinischen Revolutionärs, hg. v. A. Franke, Berlin.

L'Ordonannce Cabochienne 26-27. mai 1413 (1891), hg. v. A. Coville, Paris.

Oresme, N. (1970), Le Livre de Politiques d'Aristote, hg. v. A. Douglas Menut, in: Transactions of the American Philosophical Society, New Series, vol. 60, part 6, 1970.

Pisan, Chr. de (1936), Le Livre des fais et bonnes meurs du sage roy Charles V, hg. v. S. Solente, 2 Bde., Paris.

Pizan, Chr. de (1998), Le Livre du corps de policie, hg. v. A. J. Kennedy, Paris. 


\section{Sekundärliteratur}

Autrand, Fr. (1981), Naissance d'un grand corps de l'État, Paris.

Bell, D. M. (1955), Étude sur le Songe du Vieil Pèlerin de Philippe de Mézières, Genève.

Bulst, N. (2000), Stände und Widerstand. Die Reformvorstellungen der Generalstände von 1413 und die révolte cabochienne, in: Heinig, P.-J. (Hg.), Reich, Regionen und Europa in Mittelalter und Neuzeit, Berlin, S. 115-132.

Cazelles, R. (1962), Une exigence de l'opinion depuis Saint-Louis: la réformation du royaume, in: Annuaire-Bulletin de la Société de L'Histoire de France, 1962-1963, S. 91-99.

Contamine, Ph. (1986), Le vocabulaire politique en France à la fin du Moyen Age: l'idée de réformation, in: J.-Ph. Genet/B. Vincent (Hg.), État et Église dans la genèse de l'État moderne, Madrid, S. $145-156$

Diestelkamp, B. (1995), Rechtsfälle aus dem alten Reich, München.

Eberhard, W. (1985), „Gemeiner Nutzen“ als oppositionelle Leitvorstellung im Spätmittelalter, in: Renovatio et Reformatio. FS für Ludwig Hödl zum 60. Geburtstag, hg. v. M. Gerwig und G. Ruppert, Münster, S.195-214.

Gauvard, Cl. (1997), Le jugement entre norme et pratique: le cas de la France du Nord à la fin du Moyen Age, in: Österreichische Akademie der Wissenschaften, philosophisch-historische Klasse (Hg.), Forschungen des Instituts für Realienkunde des Mittelalters und der Frühen Neuzeit, Diskussionen und Materialien Nr.2, Wien, S. 27-38.

Honsell, Th. (1978), Gemeinwohl und öffentliches Interesse im klassischen römischen Recht, in: ZSRG (RA) 95 (1978), S. 93-137.

Köbler, G. (1986 $\left.{ }^{4}\right)$, Juristisches Wörterbuch, München.

Kennnedy, A. J. (1998), Einleitung zu Christine de Pizan, Le Livre du corps de policie, Paris.

Krynen, J. (1989), Un exemple de critique médiévale des juristes professionnels: Philippe de Mézières et les gens du Parlement de Paris, in: Histoire du droit social, Mélanges en hommage à Jean Imbert, Paris, S. 87-111.

Krynen, J. (1993), L'Empire du roi, Paris.

Leyte, G. (1996), Domaine et domanialité publique dans la France médiévale, Strasbourg.

Miethke, J. (2000), De potestate papae, Tübingen.

Müller, H. (1990), Die Franzosen, Frankreich und das Basler Konzil, 2 Bde., Paderborn, München.

Naegle, G. (1999) Französische Städte im späten Mittelalter - Verfassung und Verhältnis zur Zentralgewalt, Dissertation 2 Bde., Gießen, i.E.

Ranke, L. v. (1868), Deutsche Geschichte im Zeitalter der Reformation, Bd.6, Leipzig.

Richard, J. (1993), „Philippe de Mézières“, in: Lexikon des Mittelalters, Bd.6, München, Zürich, Sp. $592 \mathrm{f}$.

Stolleis, M. (1988), Geschichte des Öffentlichen Rechts in Deutschland, Bd.1, München.

Thoss, D. (1991), „Jouvenel des Ursins“, in: Lexikon des Mittelalters, Bd.5, München, Zürich, Sp. 640 . 
